\title{
Clinical Epidemiology of Paediatric Surgical Admissions and Mortality - A Single Centre Retrospective Study Over last 15 Years
}

\author{
Mahesh Vishwakarma ${ }^{1}$, Brajesh Lahoti ${ }^{2}$, Ashok laddha ${ }^{3}$ \\ ${ }^{1}$ Assistant Professor, Department of Surgery, Amaltas Institute of Medical Sciences, Banger, Dewas (MP), ${ }^{2}$ Professor, \\ Department of Surgery, MGM Medical College, Indore, ${ }^{3}$ Assistant Professor, Department of Surgery, MGM Medical College, \\ Indore, India
}

Corresponding author: Dr. Brajesh Lahoti, Professor, Department of Surgery, MGM Medical College, Indore, India

DOI: http://dx.doi.org/10.21276/ijcmsr.2018.3.4.28

How to cite this article: Mahesh Vishwakarma, Brajesh Lahoti, Ashok laddha. Clinical epidemiology of paediatric surgical admissions and mortality - a single centre retrospective study over last 15 years. International Journal of Contemporary Medicine Surgery and Radiology. 2018;3(4):D122-D125.

\section{A B S T R A C T}

Introduction: In developing countries, surgical diseases accounts for a major cause of neonatal mortality. Study aimed to evaluate the spectrum of paediatric surgical admissions with a comparative analysis of outcome with special reference to neonates during the time period from January 1997 to December 2011.

Material and Methods A retrospective analysis was conducted for children aged 12 years and below who were admitted during the period from 1997 to 2011. The number of admissions was grouped in 10 diagnostic categories and incidence and mortality in each category was evaluated. Mortality data were further analyzed and presented using mortality rate, proportional mortality rate and case fatality rate. Results of the study were projected with a comparison to results of similar studies in the different parts of the world.

Results: During the 15 year period, a total of 18,721 patients were admitted into the paediatric surgery division of the Department of Surgery, M.Y.Hospital Indore. The majority of patients fell into three major diagnostic categoriesGastrointestinal (31\%), Trauma (19\%), and Urogenital (14\%). The most common diagnosis with which neonates presented turned out to be Anorectal Malformations (25\%), oesophageal atresia with or without tracheoesophageal fistla (21\%) being the second most common. As a single diagnosis, over last 15 years, gastrointestinal diseases were the leading cause of surgical deaths (avg.PMR 55\%). TOF is the leading cause in the Gl category accounting for $25 \%$ of the deaths. Gastroschisis being the most fatal disease with an average CFR of $78 \%$. Incidence of Anorectal malformations and tracheoesophageal fistula was higher in this part of the country in comparison to other parts of the world.

Conclusion: Prenatal counselling should be an essential component of paediatric surgical practice and should be ensured in the training programme for future paediatric surgeons. Early recognition, risk stratification of the baby and timely referral to higher paediatric surgery units is the way forward to achieve parity with the international standards.

Key words: Tracheoesophageal Fistula, Anorectal Malformation, Proportional Mortality Rate and Case Fatality Rate

\section{INTRODUCTION}

Current data are grossly lacking on many fronts like spectrum of surgical conditions, mortality and morbidity figures and the burden of paediatric surgical diseases on our health systems. Incomplete information has made it difficult to define an appropriate role for paediatric surgery in this region of the country and to assess the impact of surgical disease on child health. Hospital admission data can be a valuable tool for assessing the epidemiology of diseases within populations. With determined, systematic and wideranging data collection, we can develop substantial insight of paediatric surgical disease trends and mortality. Our hospital is one of the 5 tertiary health institutions in the state. The hospital receives referrals for most elective and emergency surgical conditions from all over the state. This study demonstrates the extent and spectrum of paediatric surgical pathology in this part of India and highlights the need for paediatric surgical care in developing countries. ${ }^{1,2}$ Study aimed to evaluate the spectrum of paediatric surgical admissions with a comparative analysis of outcome with special reference to neonates during the time period from January 1997 to December 2011.

\section{MATERIAL AND METHODS}

A retrospective review of medical records of all patients admitted to Paediatric surgery unit, Department of surgery, MY Hospital, Indore was conducted from 1997 to 2011. The total number of admissions was grouped in 10 diagnostic categories and incidence and mortality of each category was 
evaluated.

Mortality data were further analyzed and presented using mortality rate, proportional mortality rate and case fatality rate. Results of the study were projected with a comparison to results of similar studies in the different parts of the world. Paediatric surgical centres identified through the PAPSA network were contacted and invites to participate. Those that consented to take part were asked to document all trauma admissions to their paediatric surgical service over a 1-month time period. Each participating centre was required to register the study according to their local institutions policy. Trauma is a broad term, encompassing any harm resulting from injury, accident or assault. For the purpose of this study, a paediatric trauma admission was defined as any child being referred to the paediatric surgery service as a result of attending for medical care after a trauma, either via the emergency department or through triggering a trauma call. Data were collected using a specially designed data capture form within MS excel indicating the required information. Information recorded included basic epidemiological data, specifically gender and age, mechanism of injury, injuries sustained, management of injuries, length of hospital stay, in-hospital morbidity and mortality. Morbidity was defined as any ongoing medical problem, disease or disability relating to the injury that persisted after treatment or that occurred as an iatrogenic complication following treatment of the injury. This was assessed at time of discharge or at the30-day follow-up if the patient remained an inpatient.

\section{RESULTS}

The number of paediatric patients coming to our centre has doubled itself in the past 15 years and this rise of total admissions is categorically seen in neonates with a more than threefold rise.

In above distribution, majority of admission for Gastrointestinal (31.6\%), followed by Trauma (19.3\%) and Urogenital (14.4), where as lowest admission in Neoplasm (1.5\%) (table-1).

Distribution of neonatal admission seen in highest in ARM (25\%), followed by AO with TOF (21\%), Neural tube defects (11\%), NEC/Perforation peritontitis (5.6\%) where as lowest in Choanal atresia (0.18\%) and Multicystic Kidney (0.2\%) (Table-2).

\begin{tabular}{|l|c|c|}
\hline Category & Total Admission & \% of Total \\
\hline 1. Gastrointestinal & 5920 & 31.6 \\
\hline 2. Trauma & 3620 & 19.3 \\
\hline 3. Urogenital & 2710 & 14.4 \\
\hline 4. Infections & 1654 & 8.8 \\
\hline 5. Neurosurgical & 1611 & 8.6 \\
\hline 6. Burn & 1023 & 5.4 \\
\hline 7. Head and Neck & 694 & 3.7 \\
\hline 8. Respiratory & 514 & 2.7 \\
\hline 9. Neoplasm & 291 & 1.5 \\
\hline 10. Others & 684 & 3.6 \\
\hline \multicolumn{2}{|c|}{ Table-1: Distribution of all Paediatric admissions in 10 } \\
\multicolumn{2}{|c|}{ categories } \\
\hline
\end{tabular}

\begin{tabular}{|l|c|c|}
\hline Neonatal Disease & Total Admission & \% of Total \\
\hline ARM & 672 & 25 \\
\hline AO with TOF & 569 & 21 \\
\hline Gastroschisis & 84 & 3.1 \\
\hline Omphalocele & 69 & 2.5 \\
\hline CDH & 28 & 01 \\
\hline Hirshsprung disease & 57 & 2.1 \\
\hline Intestinal Atresia & 117 & 4.3 \\
\hline NEC/Perforation peritontitis & 152 & 5.6 \\
\hline Intestinal Obstruction & 137 & 05 \\
\hline Malrotation & 20 & 0.7 \\
\hline Cong. HPS & 22 & 0.8 \\
\hline Congenital umbilical hernia & 15 & 0.5 \\
\hline Biliary atresia & 04 & 0.14 \\
\hline Neural tube defects & 300 & 11 \\
\hline Hydrocephalus & 54 & 02 \\
\hline Cleft lift and palate & 39 & 1.4 \\
\hline Exostrophy bladder & 31 & 1.14 \\
\hline Posterior urethral valve & 30 & 1.1 \\
\hline Infection & 67 & 2.4 \\
\hline Sacro. Teratoma & 24 & 01 \\
\hline PUJ Obstruction & 14 & 0.5 \\
\hline Cystic Hygroma & 12 & 0.4 \\
\hline Multicystic Kidney & 08 & 0.2 \\
\hline Choanal atresia & 2703 & 0.18 \\
\hline Others & & 6.94 \\
\hline Total & 100 \\
\hline & & \\
\hline
\end{tabular}

Table-2: Distribution of total neonatal admissions

\begin{tabular}{|l|l|l|l|}
\hline & KMC & JRH & MYH, INDORE \\
\hline OA/TOF & $2.1 \%$ & $2.1 \%$ & $21 . \%$ \\
\hline ARM & $4.7 \%$ & $9.5 \%$ & $25.73 \%$ \\
\hline NEC & $10.2 \%$ & $0 \%$ & $5.6 \%$ \\
\hline Omphalocele & $2.1 \%$ & $2.1 \%$ & $2.5 \%$ \\
\hline Gastroschisis & $5.5 \%$ & $0.9 \%$ & $3.1 \%$ \\
\hline Hirshsprung ds. & $4.5 \%$ & $10.2 \%$ & $2.1 \%$ \\
\hline Int. Atresia & $3.7 \%$ & $4.5 \%$ & $4.3 \%$ \\
\hline CDH & $2.4 \%$ & $0.4 \%$ & $1 \%$ \\
\hline \multicolumn{4}{|l|}{ Table-3: Comparison of some neonatal surgical diseases } \\
\hline
\end{tabular}

\begin{tabular}{|l|c|c|}
\hline & MYH, Indore & BHU, Varanasi \\
\hline ARM & $6 \%$ & $21 . \%$ \\
\hline TOF & $34 \%$ & $25.73 \%$ \\
\hline Hirshsprung & $21 \%$ & $5.6 \%$ \\
\hline Gastroschisis & $60 \%$ & $2.5 \%$ \\
\hline Neurosurgical Disease & $4 \%$ & $3.1 \%$ \\
\hline \multicolumn{2}{|l|}{ Table-4: Comparison of CFR in two different regions of country } \\
\hline
\end{tabular}

\section{DISCUSSION}

A wide spectrum of surgical conditions affects Indian children. Majority of the patients, however, fell into three major diagnostic categories-Gastrointestinal, Trauma, and Urogenital. In our series, these three diagnostic categories accounted for almost $64 \%$ of paediatric surgical admissions (table 1). Gastrointestinal diseases including congenital malformations (eg. TOF and ARM) were responsible for 
the largest number of admissions (31\%). Trauma (19\%) was the second most common category including head injury, polytrauma, foreign body ingestion etc. Urogenital disease (14\%) was the third major category involved. Infections (9\%) and Burn (5.4\%) were other common categories.

While evaluating the neonates congenital anomaly was found to be the most common cause of neonatal admissions. Congenital anomaly of the gastrointestinal system (59.3\%) has the highest occurrence, followed by that of the central nervous system (13\%) (table-2). It was observed that TOF, anorectal malformations and Neural tube defects were the three most frequent diagnoses encountered forming the bulk of admissions over the past 15 years. The most common diagnosis with which neonates presented to the department of Paediatric surgery turned out to be Anorectal Malformations.

The average percentage contribution of ARM was 25.73\%. Despite this, a particular rise in the number of cases was observed in the time period from 1998 to 2001.

Even though TOF remained to be one of the most common disorder that the neonates presented with yet the percentage contribution to the total admission has more or less remained the same with average of $21.10 \%$. Exception to this observation was the year 1997 when the percentage of TOF (28.5\%) exceeded that of the ARM (23.5\%).

NEC/Perforation peritonitis shows a rise in the number of admissions from the year 1997(2\%) to 2003(10\%). From the year 2004 the incidence of the disease seems to decrease which constitutes 3\% of neonates in the year 2011 .

Neurosurgical disease including Hydrocephalus, Meningomyelocele also constitutes a major part of neonatal admissions with an average of $12 \%$ of total admissions.

We compared our results (incidence) with a comparative study of neonatal surgical admissions between two linked surgical departments in Africa and Europe. The study was conducted at the Kilimanjaro Christian Medical Centre (KCMC), Tanzania and the John Radcliffe Hospital, UK (JRH) between the time periods from 2003-2005 (table3). ${ }^{3}$ In our study neonates presenting as NEC with intestinal perforation were included in the category NEC, which is a common disease of premature babies. The incidence of NEC at JRH (10.2\%) was double of that MYH, Indore (5.6\%).

Statistically significant difference was observed in the percentages of admissions for OA with TOF from $\mathrm{MYH}$, Indore in comparison to that of JRH and KCMC with a much higher incidence in MYH, Indore.

The incidence of Hirshsprung disease was much less in Indian subcontinent in comparison to other two regions.

The spectrum of neonatal surgical admissions in these institutions varied. We believe this is attributable to availability of antenatal diagnosis, primary health-care and transport facilities as well as genetics.

Over the last decade there has been a significant fall in the incidence of ARM which constitutes $35 \%$ of total neonatal admissions in the year 2001, through some variations it came down to $19.5 \%$ in the year 2011 . TOF appears to be the second most common neonatal disease; though the recent trends suggest that the incidence of TOF parallels to that of the ARM.
As a single diagnosis, over last 15 years, Gastrointestinal diseases were the leading cause of surgical deaths (avg. PMR 55\%). This is evident that TOF, as a single disease entity contributes $25 \%$ of paediatric surgical deaths adversely affecting the overall survival rate at our centre. Other GI diseases including congenital diseases (ARM, Gastroschisis, Omphalocele, Hirshsprung disease etc.) and acquired (Perforation peritonitis, Intestinal obstruction) constitute $30 \%$ of the deaths.

Burn was the second leading cause of deaths in paediatric patients which constitutes $15 \%$ of total deaths followed by Trauma (10\%).

Neurosurgical diseases (including congenital hydrocephalus, NTD and TBM with hydrocephalus) are also a major cause of death with an average proportional mortality rate of $8 \%$.

- A mong the major killing diseases maximum Case fatality rate (which indicates the killing power of a disease) is seen in gastrointestinal categories. Gastroschisis being the most fatal disease with an average CFR of $78 \%$. TOF carries a CFR of $65 \%$ which appears to be a relatively common disease with high mortality. Omphalocele patients have an average CFR of $53 \%$ which indicates better survival than gastroschisis.

We have an average mortality rate of $8.5 \%$ in last 15 years with highest mortality rate of $12 \%$ in the year 1998 and a lowest mortality rate of $6.7 \%$ in the year 2009 .

- On comparing the results of the present series with series of A.N. Gangopadhyay et $\mathrm{al}^{4}$ in the university hospital BHU Varanasi, India over year 1996 - 2006, we conclude that Survival rate among TOF patients at MYH, Indore was low (35\%) as compared with BHU, another tertiary centre in the different part of the country (66\%) (table-4).

R.K.Tandon et $\mathrm{al}^{5}$ had earlier reported better survival in TOF patients as per Waterson criteria was $100 \%$ in group A, 83\% in group B and 22\% in group C in a study period from 2004 to 2006 at Lucknow, India in contrast to MYH Hospital Indore(35\%) Spitz et $\mathrm{al}^{6}$, London, UK, reported better results, survival rates according to Waterson classification system was $100 \%$ for class A, $86 \%$ for class B and $73 \%$ for

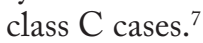

In a Population based cohort study of all live born infants with gastroschisis ${ }^{8}$ born in the United Kingdom and Ireland from October 2006 to March 2008 the overall case fatality was $4 \%$ which is significantly low than our series (78\%).

The results of this study provide valuable insight into the surgical conditions that affect children living in this region of the country.

Availability of antenatal diagnosis and subsequent delivery in an institution with specialised paediatric surgical unit with neonatal intensive care facilities, primary health-care, availability of transport facilities and possibly a different threshold for presentation all contributed to the better survival of surgical neonates in developed countries..$^{9,10}$

\section{CONCLUSION}

Hence, we concluded significant improvement has been achieved in the outcome of paediatric surgery over the few years. This development has to continue further to achieve parity with international standards. Prenatal counselling 
should be an essential component of paediatric surgical practice and should be ensured in the training programme for future paediatric surgeons. Healthcare structure, access to paediatric surgery and trauma prevention strategies may account for these differences. Early recognition, risk stratification of the baby and timely referral to higher paediatric surgery units is the way forward.

\section{REFERENCES}

1. Goodman LF, St-Louis E, Yousef Y et al. The global initiative for children's surgery: optimal resources for improving care. Eur J Pediatr Surg 2017;2(1):12-18.

2. Bradshaw CJ, Lakhoo K, Ameh E et al. A day in the life of a paediatric surgeon: a PAPSA research study. Ann Pediatr Surg 2016;7(3):13-19.

3. B. Nandi C. Mungongo K. Lakhoo: A comparison of neonatal surgical admissions between two linked surgical departments in Africa and Europe, Pediatr Surg Int. 2008; 24(8):939-42.

4. Gangopadhyay AN, Upadhyaya VD, Sharma SP: Neonatal surgery: a ten year audit from a university hospital. Indian J Pediatr. 2008; 75(10):1025-30.

5. Tandon RK, Sharma S, Sinha SK, Rashid KA, Dube R, Kureel SN, Wakhlu A, Rawat JD: Esophageal atresia: Factors influencing survival - Experience at an Indian tertiary centre, J Indian Assoc Pediatr Surg. 2008;13(1):2-6.

6. Spitz L, Kiely E, Brereton RJ: Esophageal atresia: five year experience with 148 cases.,J Pediatr Surg. 1987;22(2):103-8.

7. Moonesinghe SR, Mythen MG, Das P, et al. Risk stratification tools for predicting morbidity and mortality in adult patients undergoing major surgery: qualitative systematic review. Anesthesiology 2013;119(5):959-81.

8. Marian Knight: Gastroschisis: one year outcomes from national cohort study, BMJ 2011; 343(3):d6749

9. Abelha FJ, Castro MA, Landeiro NM, et al. Mortality and length of stay in a surgical intensive care unit. Rev Bras Anestesiol 2006;56(2):34-45.

10. Glance LG, Lustik SJ, Hannan EL, et al. The Surgical Mortality Probability Model: derivation and validation of a simple risk prediction rule for noncardiac surgery. Ann Surg 2012;255(3):696-702.

Source of Support: Nil; Conflict of Interest: None

Submitted: 12-10-2018; Accepted: 14-11-2018; Published online: 25-12-2018 